\title{
The biological and clinical significance of stromal-epithelial interactions in breast cancer
}

\author{
Robert McCuaig ${ }^{1}$, Fan $\mathrm{Wu}^{1}$, Jenny Dunn ${ }^{1}$, Sudha Rao ${ }^{1}$ and \\ JANE E. DAHLSTROM ${ }^{2}$ \\ ${ }^{1}$ Health Research Institute, Faculty of Education, Science, Technology and Mathematics, \\ University of Canberra, Bruce, and ${ }^{2}$ Anatomical Pathology, ACT Pathology and Australian \\ National University Medical School, The Canberra Hospital, Woden, ACT, Australia
}

\begin{abstract}
Summary
There is evidence that an aberrant tumour microenvironment (TME) facilitates cancer development, progression, and responses to treatment. While many of the mechanisms underlying the phenotype and cancer-promoting behaviour of the TME are unknown, epigenetic mechanisms in cancer cells and the TME are thought to play important roles. As a result, cancer profiling strategies for drug and biomarker development require a thorough understanding of both the epithelial tissue compartment and the TME. This review discusses recent advances in our understanding of how cancer epithelial cells interact with their microenvironment and how this knowledge can be exploited clinically.
\end{abstract}

Key words: Breast cancer; epigenetics; tumour microenvironment; cancer stem cells; epi-drugs; cancer associated fibroblasts; DNA methylation; histone.

Received 18 August, revised 3 October, accepted 5 October 2016 Available online 29 December 2016

\section{INTRODUCTION}

Breast cancer tissues contain genetic and epigenetic changes that result in altered epithelial cell structure and function. Epigenetic regulation is defined as any heritable modifications in gene expression and chromatin structure caused by alterations that do not involve the primary nucleotide sequence. ${ }^{1,2}$ Epigenetic changes include DNA methylation, post-translational modifications of histone proteins, nucleosomal positioning, incorporation of histone variants, and the action of non-coding RNAs [such as micro (mi)RNAs]. ${ }^{3}$ The 'classical' epigenetic effect occurs when epigenetic silencing of one allele acts in concert with an inactivating mutation in the opposite allele, resulting in total allelic loss; for example, hypermethylation and deletion of the $B R C A 1$ promoter in sporadic breast cancer. ${ }^{4}$

The tumour microenvironment (TME) also represents an important source of epigenetic regulation of the epithelial compartment in breast cancer. As well as harbouring malignant cells, the TME contains cells of mesenchymal and haematopoietic origin and non-cellular components. ${ }^{5}$ Cells of mesenchymal origin in the TME include fibroblasts, myofibroblasts, mesenchymal stem cells (MSCs), adipocytes, and endothelial cells, while cells of haematopoietic origin include lymphoid cells [T cells, B cells, and natural killer (NK) cells] and myeloid cells [macrophages, neutrophils, and myeloid-derived suppressor cells (MDSCs)]. The non-cellular component is the extracellular matrix (ECM) formed by the basement membrane and interstitial matrix (consisting of collagens, proteoglycans, and glycoproteins) (Fig. 1).

The TME also has an important metabolic $\left(\mathrm{pH}, \mathrm{PO}_{2}\right.$, glucose, glutamine, lactate) and chemical (e.g., nitric oxide) environment. ${ }^{6}$ This is further discussed by Simmons et al. in this issue. ${ }^{7}$ Experimental modelling has shown that epigenetic cross-talk between cells in the TME drives the efficiency of cancer formation, the rate of cancer growth, the extent of invasion, the ability of cancers to metastasise, and their response to treatments.

\section{CANCER-ASSOCIATED FIBROBLASTS IN THE TME}

What are cancer-associated fibroblasts?

Fibroblasts are generally the most abundant cell type in the TME. A subpopulation of fibroblasts known as cancerassociated fibroblasts (CAFs) is thought to be of critical importance in cancer initiation, progression, survival, metastasis, and invasion via the secretion of various growth factors, cytokines, and chemokines and the degradation of ECM proteins. 9,10 The origins of CAFs in breast cancer stroma are diverse. ${ }^{10}$ The vast majority are thought to arise from normal fibroblasts, and breast cancer cells are known to induce epigenetic changes in normal fibroblasts that transform them into CAFs. For instance, Tyan et al. ${ }^{11}$ showed that breast cancer cells can induce hepatocyte growth factor (HGF) secretion by CAFs to enhance tumorigenesis and that when normal fibroblasts were cultured with the breast cancer cell line MDA-MB-231 they secreted HGF and adopted a $\mathrm{CAF}$ phenotype. In another example, the MCF-7 breast cancer cell line was found to reduce caveolin-1 (Cav-1) expression (a CAF biomarker) in normal fibroblasts, resulting in phenotype switching to CAFs and increased expression of CAF-associated markers. ${ }^{12}$

CAFs also arise when epithelial cells undergo epithelialmesenchymal transition (EMT), from bone marrow-derived stem cells that have undergone EMT, or from transdifferentiated breast tissue cells such as pericytes, adipocytes, or smooth muscle cells (Fig. 2). ${ }^{13,14}$ The CAF profile differs 


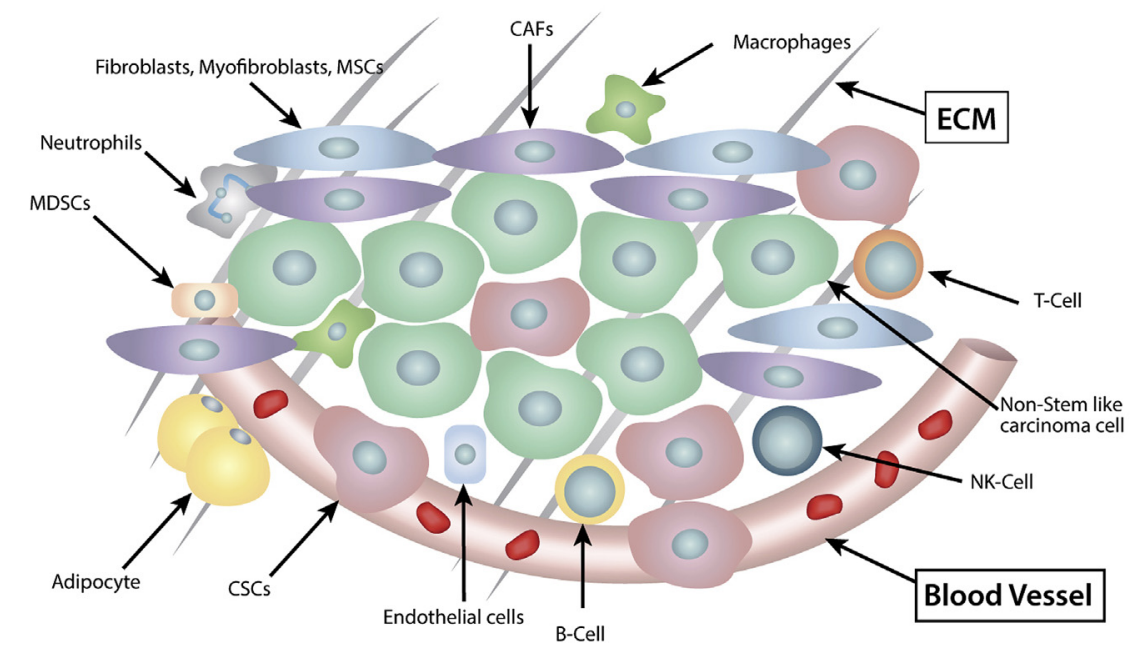

Fig. 1 Components of the tumour microenvironment (TME). The TME is a complex mixture of not only carcinoma cells but also many cells of different lineages and extra-cellular material. The cellular component includes cells of mesenchymal origin [fibroblasts, cancer-associated fibroblasts (CAFs), myofibroblasts, mesenchymal stem cells (MSCs), adipocytes, and endothelial cells] and those of haematopoietic origin: lymphoid cells [T cells, B cells, and natural killer (NK) cells] and myeloid cells (macrophages), neutrophils, and myeloid-derived suppressor cells (MDSCs). The non-cellular component is the extracellular matrix (ECM). The TME is an important epigenetic regulator of the epithelial compartment in breast cancer that ultimately influences the cancer phenotype.

depending on the TME and breast cancer subtype. In general, CAFs highly express $\alpha$-SMA, p53, podoplanin, CD10, fibroblast activation protein (FAP), matrix metalloproteinases (MMPs), tenascin-C, and platelet-derived growth factor $(\mathrm{PDGFR} \alpha / \beta)$ and lose Cav-1 expression. ${ }^{10,12}$ Cytoskeletonand integrin signalling-associated genes are up-regulated in HER2+ breast cancers compared to triple-negative breast cancers. ${ }^{15}$ However, a universal CAF 'signature' has so far proven elusive. ${ }^{10}$

The gene expression profiles of fibroblasts from women without breast cancer have been compared to those from women with breast cancer. ${ }^{16}$ Many genes are up-regulated in CAFs compared to normal fibroblasts including growth factors [fibroblast growth factors (FGFs), hepatocyte growth factor (HGF), transforming growth factor beta (TGF- $\beta$ ), and stromal cell-derived factor 1 (SDF-1)], cytokines [granulocyte macrophage colony-stimulating factor (GM-CSF), effector cell protease receptor 1 (EPR-1)], oncoproteins (K-ras), regulators of gene expression (nuclear-encoded mitochondrial elongation factor Ts, ribosomal protein S12, and spliceosomeassociated protein SAP 145), and a variety of other genes associated with the cell cycle, cell-cell interactions, and cellcell communication. Many of these gene products are proinvasive and pro-metastatic. ${ }^{10,17}$

Breast cancer CAFs also show aberrations in DNA methylation, histone modifications, and dysregulated miRNAs. ${ }^{18}$ While all DNA is coated with methyl moieties, the DNA methylation pattern is regulated by an independent enzymatic process catalysed by DNA methyltransferases (DNMTs). In terms of DNA methylation, the DNA methylation profiles of 143 human breast tumours showed significant differences in HER2 expression and DNA methylation

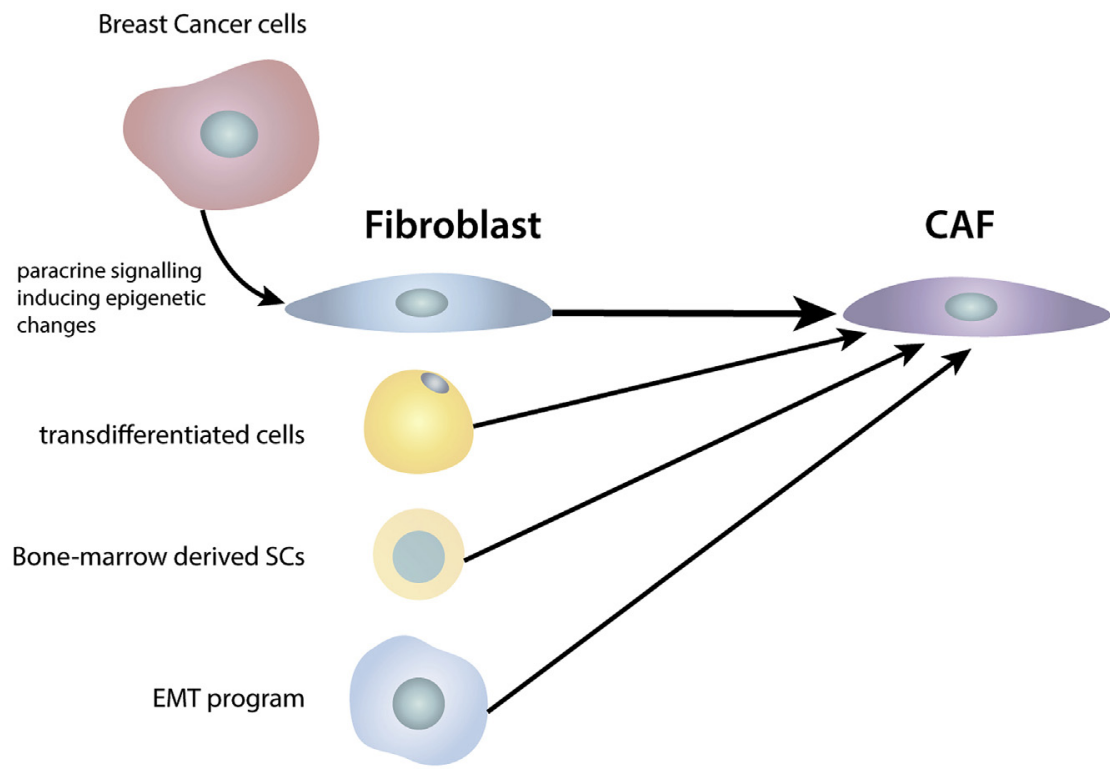

Fig. 2 Potential origins of cancer-associated fibroblasts (CAFs). There are multiple sources of CAFs, with the vast majority arising from phenotypic switching of normal fibroblasts under the influence of epigenetic signalling from breast cancer cells (non-stem-like carcinoma cells and cancer stem cells). However, CAFs can also arise from epithelial-mesenchymal transition (epithelial cells), transdifferentiated pericytes, adipocytes, or smooth muscle cells, and bone marrow-derived stem cells. These TMEinfluenced pathways induce epigenetic changes that promote a CAF profile. 
of five genes, three of which were also methylated in the tumour stroma as well as the cancer cells. ${ }^{19}$ Furthermore, methylation-specific digital karyotyping revealed epigenetic alterations in stromal fibroblasts as well as epithelial and myoepithelial cells in normal breast tissues compared to in situ and invasive carcinomas. ${ }^{18}$

DNA is normally wrapped around histones, thereby providing another important mechanism of gene regulation. Loss of histone deacetylase 1 (HDAC1) expression has been shown to increase osteopontin glycoprotein expression within the stromal compartment of invasive breast cancers, which then activates CAFs to promote tumour growth in vivo. ${ }^{20}$ MicroRNAs (miRNAs) are a class of short noncoding regulatory RNAs involved in stem cell maintenance, developmental programming, cell fate specification, and various pathologies, not least cancer. Depending on the protein targeted, miRNAs can act as either oncogenes or tumour suppressors. miRNAs have been shown to be dysregulated in breast CAFs; in particular, miR-200s are down-regulated in activated CAFs in breast cancer tissues. ${ }^{21}$

\section{Breast CAFs can induce EMT in breast cancer cells and the cancer stem cell phenotype}

The process of EMT confers mesenchymal properties on epithelial cells that are closely associated with the acquisition of aggressive traits seen in the cancer stem cell (CSC) phenotype. ${ }^{22}$ Furthermore, such CSCs have been shown to resist standard anticancer therapies. ${ }^{23}$ Several studies have shown that breast CAFs can induce an EMT signature (including increased vimentin expression and decreased Ecadherin expression) in breast cancer cells (Fig. 3). ${ }^{23-25}$ For example, non-CSCs [also known as non stem-like cancer cells (NSCs)] derived from human mammary basal epithelial cells (HMECs) maintain the ZEB1 promoter, a key regulator of EMT transition, in a bivalent chromatin configuration. ${ }^{26}$ Bivalency in this context refers to chromatin existing in a repressed state but poised for rapid transcriptional activation in response to signalling cues that favour differentiation; this enables them to switch to a stem cell-like state. In the bivalent state, the $Z E B 1$ promoter is marked with both active
(H3K4me3) and repressive (H3K27me3) histones. In response to microenvironmental signals such as TGF- $\beta$, a well-known EMT inducer, released by CAFs, the ZEBI promoter converts to an active chromatin configuration (with loss of $\mathrm{H} 3 \mathrm{~K} 27 \mathrm{me} 3$ ), ZEB1 transcription increases, and nonCSCs enter the CSC state. These CSCs can self-renew and drive tumorigenesis. Breast CSCs display a CD44+/CD24cell surface marker profile and are also known to form a subpopulation of circulating tumour cells that might give rise to metastases. CAFs, through increased expression of the chemokine CCL2 via NOTCH1-STAT3 activation, have been shown to stimulate the CSC phenotype in breast cancer cells and inhibition of CCL2 in fibroblasts in xenograft models of breast cancer abrogates this tumorigenic effect. ${ }^{27}$

De Marco et $a l .{ }^{28}$ recently showed that CAFs mediate the malignant phenotype and cancer aggressiveness through secretion of IL- $1 \beta$ into the TME and its consequent effect on breast cancer cells, as IL-1 $\beta$ drives a pro-tumorigenic inflammatory phenotype via the IL1R1 receptor on cancer cells. The G-protein oestrogen receptor (GPER) triggers upregulation of IL-1 $\beta$ and ILIR1 expression on CAFs and breast cancer cells, respectively, to promote breast cancer cell migration and invasion. ${ }^{28}$ The relationship between EMT and epithelial CSCs indicates that EMT is thus particularly dangerous since, by imparting mesenchymal traits to carcinoma cells, EMT can generate properties associated with high-grade malignancy including motility, invasion, resistance to apoptosis, and metastasis. ${ }^{22}$

\section{CAFs and their effect on other components of the microenvironment}

CAFs are also known to mediate a tumour-enhancing inflammatory profile driven by $\mathrm{NF}-\kappa \mathrm{B}$, which promotes angiogenesis and tumour growth via the release of growth factors (FGFs, HGF, TGF- $\beta$, SDF1), cytokines (CXCL12, IL-6), and hormones (such as oestrogen) in the TME. ${ }^{29-33}$ CAFs can attract immunosuppressive cells (for example, FOXP3 T cells and myeloid-derived suppressor cells) to the TME that inhibit $\mathrm{T}$ cell function and suppress adaptive immunity and natural killer cell function. ${ }^{34,35} \mathrm{CAF}$ elimination

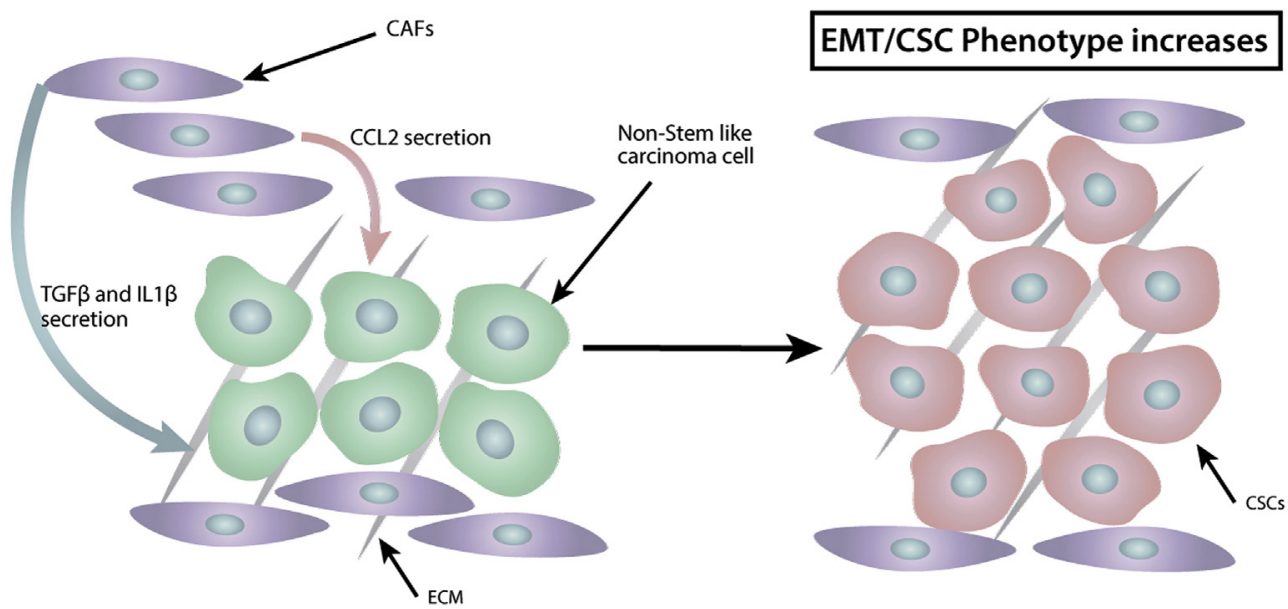

Fig. 3 The role of cancer-associated fibroblasts (CAFs) in breast cancer epithelial-mesenchymal transition (EMT) and the cancer stem cell (CSC) phenotype. As shown in Fig. 2, CSCs can induce epigenetic changes in fibroblasts to create a CAF phenotype. In this context, CAFs can induce a CSC profile in non-stem-like epithelial carcinoma cells through secretion of TGF- $\beta$ and IL-1 $\beta$ into the tumour microenvironment (initiating EMT) and/or via increased expression of the chemokine CCL2 by CAFs interacting with carcinoma cells to induce transition of the non-stem-like carcinoma cells (NSCs) to a more mesenchymal state. Both of these pathways increase the acquisition of the mesenchymal CSC phenotype that favours breast cancer cell migration, invasion, and resistance to therapy. 
has been shown to reduce tumour-associated macrophages and myeloid-derived suppressor cell recruitment to tumours. ${ }^{36}$ CAFs have also been shown to depress the Th1 immune response by suppressing Th1 cytokines and enhancing an immunosuppressive Th2 cytokine signature and tumour growth in CAF-knockout cancer metastasis models in vivo. ${ }^{36}$

\section{OTHER MESENCHYMAL COMPONENTS OF THE TME AND BREAST CANCER}

\section{Adipocytes and endothelial cells in the TME}

Other cells of mesenchymal origin in the TME include adipocytes and endothelial cells. The concept that adipocytes participate in cancer initiation, growth, and metastasis is now called 'adiponcosis'. ${ }^{37}$ For example, the release of CCchemokine ligand 5 (CCL5) by adipocytes has been shown to promote the motility and invasion of triple-negative breast cancer cells. ${ }^{38}$ The secretion of factors such as leptin and interleukin 6 (IL-6) by adipocytes has been shown to be important in inducing breast cancer cell EMT and activating CSC pathways. ${ }^{39}$ Furthermore, adipocytes have been shown in vitro and in vivo to participate in a highly complex cycle whereby breast cancer cells modify the phenotype of peritumoral adipocytes that in turn modify the cancer cell phenotype to promote cancer progression. ${ }^{40}$

In addition to the accepted roles of tumour blood and lymphatic vessels as conduits for blood supply and tumour dissemination, these vessels have also been shown to play an important role in cancer cell crosstalk via molecules secreted by the blood (BEC) and lymphatic (LEC) endothelial cells (also called angiocrine and lymphangiocrine factors). BECcancer cell crosstalk can induce stem cell-like properties and EMT in cancer cells similar to CAFs. ${ }^{41}$ Lee et al. ${ }^{42}$ showed that triple-negative breast tumours induced LECs to secrete CCL5, which recruits CCR5-expressing cancer cells into the lymphatic system, thereby promoting lymph node metastasis.

\section{Haematopoietic cells of the TME}

The cells of haematopoietic origin in the TME include lymphoid cells (T cells, B cells, and NK cells) and myeloid cells (macrophages, neutrophils, and MDSCs). A detailed discussion of the role of tumour-infiltrating lymphocytes is the subject of a number of detailed reviews including in this edition and will not be discussed further. ${ }^{43,44}$

MDSCs are a heterogeneous population of immature myeloid cells that inhibit innate and adaptive immunity and suppress various immune cells such as T cells, dendritic cells, and NK cells and stimulate immune modulators such as Th2 $\mathrm{T}$ cells, $\mathrm{T}$ regulatory cells (Treg), and tumour-associated macrophages (TAMs). Increased MDSCs are closely correlated with increased tumour burden and the duration of breast cancer. $^{45}$

NK cells are a major component of the antitumor immune response and are involved in controlling tumour progression and metastasis in animal models. Mamessier et al. ${ }^{46}$ showed that NK cell dysfunction causes human breast cancer progression. Breast cancer cells appear to alter NK cell function by modulating their surface receptors, and several stromaderived factors including TGF- $\beta 1$ in the TME are involved in a tumour-induced reduction in normal NK cell function. ${ }^{46}$

\section{The ECM of the TME}

The ECM constitutes the non-cellular component of the TME and includes the basement membrane and interstitial matrix (consisting of collagens, proteoglycans, and glycoproteins). There is good evidence to suggest that proteoglycan/glycosaminoglycan expression levels and fine structures are involved in breast cancer growth, invasion, and metastasis. For example, the proteoglycan versican has been shown to stimulate mesenchymal-epithelial transition (MET) and increase breast cancer cell proliferation at metastatic sites. ${ }^{47}$ DNA methylation also plays an important role in regulating collagens in cancer. ${ }^{48}$ Chernov et al. ${ }^{49}$ showed in MCF-7 breast cancer cells that certain collagen genes are epigenetically silenced by H3K27me3 repression, which changes the ECM composition to an invasion-promoting collagenenriched matrix. $^{49}$

The roles of various elements of the TME and their role in breast cancer are summarised in Fig. 4.

\section{CONTRIBUTION OF THE TME TO METASTASIS IN BREAST CANCER}

Metastasis is an inefficient process. For most breast cancers only a minority of cancer cells successfully spread, colonise and grow at a distant site. ${ }^{50}$ Metastasis is also dependent on the metastatic niche, which describes the specialised microenvironment that supports metastatic events and the environment at distant metastatic sites. ${ }^{51}$ The metastatic niche model proposes two mechanisms by which successful metastasis might occur: through either a pre-existing niche or an induced metastatic niche. A pre-existing niche is a site that supports a pre-existing normal, specific physiological function that is then co-opted or hijacked by a metastatic cancer cell to aid its survival in the new TME such as within bone marrow. ${ }^{52}$ In contrast, an induced niche is present when there have been changes to the nature of the microenvironment's ECM and component cells that are permissive to colonisation by metastatic cells. ${ }^{52,53}$

The TME also plays a critical role in regulating metastatic events in breast cancer. ${ }^{52,54,55}$ Metastasis is not a single event but rather a complex series of events that includes the invasive tumour cell growth, ECM degradation, and invasion into the lymph or circulatory system. As noted above, tumour cells must undergo EMT to acquire an invasive and mesenchymal phenotype, and CAFs - a primary component of the breast cancer stroma - are significant mediators of EMT and metastasis. ${ }^{56}$ However, this is not their sole function, as CAFs can also enhance invasion through active degradation of the ECM via a diverse array of mediators including upregulation of palladin (a cytoskeletal protein) and proteolytic enzymes. ${ }^{57}$ CAFs can also play a direct role in cancer metastasis by leading the actual invasion and metastasis of tumour cells from the site of the primary tumour. ${ }^{58}$

The niche colonised by cancer cells is an important part of the metastatic process. The ability of metastases to thrive at distant sites requires tumour cells to adapt to new and often dramatically different microenvironments. ${ }^{59}$ Work by Malanchi et al ${ }^{54}$ demonstrated in a breast cancer model that POSTN is a critical mediator expressed in primary tumour stroma and that CSCs migrating to distant sites also need to induce stromal POSTN so that the tumour cells can colonise the new site. In this respect, the invading tumour cells alter the new microenvironment to be more receptive to metastatic 


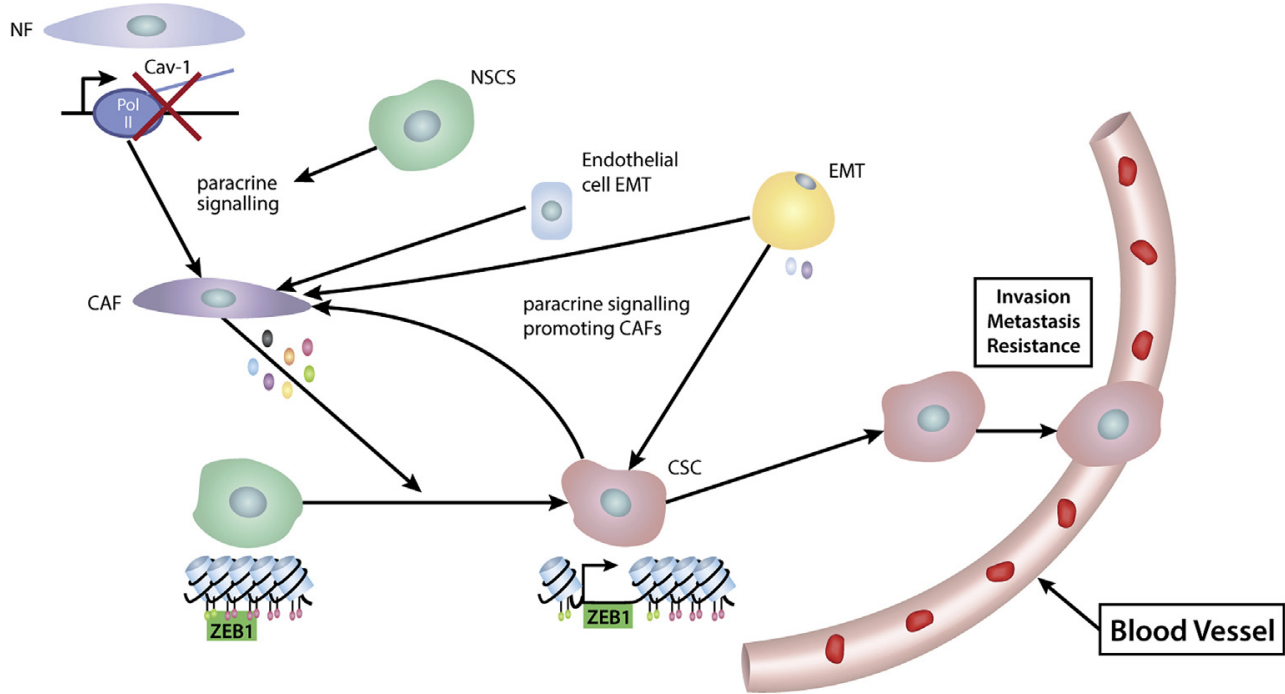

Fig. 4 The various elements of the tumour microenvironment (TME) and their role in breast cancer. Breast non-stem-like cancer cells (NSCs) can, through paracrine signalling, inhibit the expression of caveolin-1 (CAV-1) in normal fibroblasts to promote a shift to a cancer-associated fibroblast (CAF) signature. The CAFs then increase expression of a variety of mediators that enhance tumorigenesis $($ coloured balls: red $=$ HGF, blue $=$ TGF $\beta$, orange $=$ CCL2, green $=$ IL6, yellow $=$ IL $1 \beta$, purple $=$ SDF-1, black $=$ CXCL12). This drives EMT and induces the non-stem-like cancer cell ZEB1 promoter to lose its tri-methylation on H3K27 (inactive mark red balls) and retain its active mark (green balls) H3K4me3, resulting in increasing chromatin accessibility of ZEB1 and transcription and allowing entry to the CSC signature that enhances invasion, metastasis, and resistance to therapy. The new CSC can in turn induce the formation of more CAF signature cells, resulting in feedback that increases overall tumour malignancy. In addition to this pathway, CAFs can also arise from endothelial cell EMT and other transdifferentiated cells (blue square cells). Adipocytes are also involved in this TME regulation (a process known as adiponcosis), contributing to tumorigenesis via EMT differentiation into CAFs to enhance cancer growth, survival, and aggressiveness through the secretion of IL6 (pink balls) and CCL5 (light blue balls).

colonisation. ${ }^{54}$ Zhang et al. ${ }^{55}$ recently illustrated an elegant example of tumour cell adaptation to a new TME in brain metastasis, with tumour cells losing PTEN expression after metastatic invasion of the brain but not other tissues and organs. It was proposed that extracellular vesicles (EVs) containing PTEN-blocking microRNAs may have mediated this knockdown effect. EVs comprise a diverse range of structures of different cellular origin and highly variable 'cargo' and include exosomes, microvesicles, and large oncosomes, ${ }^{60}$ all of which can influence the TME. EVs can reprogram cancer cell metabolism, mediate therapeutic drug interactions, and are implicated in driving the transformation of fibroblasts and other TME cells to a CAF phenotype. ${ }^{61-63}$ EVs can in turn enhance the metastatic potential of the tumour such as up-regulating MMP-9 expression in melanoma cells and reprogramming cancer cells with enhanced metastatic potential. ${ }^{64}$ Le et al. ${ }^{65}$ showed that EVs containing miR-200 can alter gene programming and promote MET. Tumour metastasis is enhanced by the mesenchymal phenotype; however in many tumours, including breast cancer, invading cells require epithelial traits to colonise other sites. miR-200 can confer strong metastatic potential to either adjacent cells or even tumour cells at distant sites through this re-programming. ${ }^{65}$

\section{THE TME AND THERAPEUTIC RESISTANCE IN BREAST CANCER}

Despite significant improvements in outcomes for breast cancer patients over the last 20 years, drug resistance and tumour recurrences occur. The TME, and specifically CAFs, are implicated in breast cancer resistance to therapy. ${ }^{14}$ Several authors have shown that CAFs play a significant role in tamoxifen resistance through activation of the PI3K/ AKT and MAPK/ERK pathways and induction of the oestrogen receptor (ER) via the G protein-coupled ER to promote proliferation and progression of breast cancer cells. ${ }^{66-68}$ Furthermore, Yuan et al. ${ }^{69}$ demonstrated that the $G$ protein-coupled ER GFR/ERK pathway increases $\beta 1$ integrin expression to contribute to CAF-associated EMT, invasion, and tamoxifen resistance in MCF-7 breast cancer cells. ${ }^{69}$ Mao et al $^{70}{ }^{7}$ recently demonstrated that CAFs can render HER2+ breast cancer cells resistant to trastuzumab via IL-6 and the activation of multiple pathways including NF$\kappa \mathrm{B}, \mathrm{JAK} / \mathrm{STAT} 3$, and PI3K/AKT. ${ }^{70}$ Farmer et al. ${ }^{71}$ demonstrated that very desmoplastic stroma in breast cancer produces a stromal gene expression that predicts resistance to pre-operative chemotherapy. ${ }^{71}$ The TME, and in particular the CAFs and immune cells, are also thought to play a role in radiotherapy resistance. ${ }^{72}$ As various immunotherapies are introduced in breast cancer management, it is likely that both intrinsic but acquired therapeutic resistance will occur in the TME as reported in other cancers. ${ }^{73}$

\section{THERAPEUTIC TARGETING OF THE TME USING EPIGENETIC DRUGS}

The current standard treatment for breast cancer includes surgery, radiotherapy, chemotherapy and hormone therapy. The treatment given largely depends on the pathological characteristics of the breast cancer, the clinical stage of disease, and the age and general health of the patient. One of the potentially most attractive new therapies is targeting epigenetic inheritance because, in contrast to DNA mutations, these defects are passively inherited and thus must be actively maintained because they are reversible. Re-expression of genes epigenetically inactivated can suppress the disease state or sensitise to specific therapies. Small molecules that reverse epigenetic inactivation, so-called 'epi-drugs', are now undergoing clinical trials. To date, most epi-drug studies have focused on the direct treatment of the epithelial cell compartment rather than the TME. The best-studied 
epigenetic drugs are the histone modifiers. Histones are modified by histone-modifying enzymes that add or remove covalent moieties to histone proteins. ${ }^{74}$ Among these, histone acetyl transferases (HATs) act as 'writers' that transfer acetyl groups to lysine residues on histones to activate transcription, and histone deacetylases (HDACs) act as 'erasers' that remove acetyl groups to repress gene transcription. ${ }^{75}$ The term 'HDAC inhibitors' refers to a group of compounds that target this latter mechanism, and these drugs have been tested in breast cancer clinical trials. ${ }^{76,77}$ DNA methyltransferase (DNMT) inhibitors are another group of epigenetic drugs that have been shown to inhibit cell growth and work well in combination with HDAC inhibitors. Furthermore, some DNMT inhibitors have been shown to re-express functional ER in ER negative breast cancer cells in which the ER has been epigenetically silenced. ${ }^{78}$ Both DNMT and HDAC inhibitors have been shown to re-express epigenetically silenced genes in breast cancer and improve patient outcomes; however, long-term efficacy is limited and very high doses are often required, suggesting that combination strategies to enhance responses are important. ${ }^{76,79}$

Epigenetic modifying agents have been shown to 'prime' the immune system, making combination therapy with immune modulators attractive. Kim et al. ${ }^{80}$ showed that 5azacytidine (5-AZA; a DNA methyltransferase inhibitor) in combination with anti PD-1 and anti CTLA-4 monoclonal antibodies eradicated both primary and metastatic tumours in mice. ${ }^{80}$ 5-AZA has also been shown to up-regulate genes responsible for antigen processing and presenting [such as tumour-associated antigens, major histocompatibility complex proteins, co-stimulatory molecules (e.g., CD40, CD80, CD86, ICAM-1)] and death-inducing receptors that target cancer cells (e.g., FAS), causing a shift in the balance of immune inhibition towards immune activation. ${ }^{81}$

Recent studies by our laboratory (Zafar et al. $^{82}$ and Boulding et $a l .{ }^{83}$ ) have identified potential new classes of epigenetic therapeutic targets (Fig. 5). While protein kinase $\mathrm{C}$ (PKCs) have long been recognised as cytoplasmic signalling proteins, we and others have shown that PKCs belong to an emerging class of kinases that have a dual function as epigenetic enzymes. ${ }^{84-86}$ These chromatin-tethered kinases modulate chromatin structure to make it conducive for active transcription by two distinct mechanisms: (1) structural (as part of transcription complexes) and (2) enzymatic (by phosphorylating key nucleosome components, the basic unit of chromatin). ${ }^{82}$ Our findings have shown that PKC-theta (PKC- $\theta$ ) is a signalling kinase that is specifically targeted to the nucleus to function as a transcriptional regulator by tethering to the epigenome; $\mathrm{PKC}-\theta$ is also a critical molecular mediator of CSC function. This nuclear kinase mediates crosstalk between extracellular signals and the epigenome in breast CSCs in response to TGF- $\beta$ and inflammatory signals mediated via the NF- $\kappa$ B pathway in breast cancer. ${ }^{2}$ In particular, our genome-wide PKC- $\theta$ chromatin immunoprecipitation (ChIP)-sequencing analysis identified direct PKC$\theta$-dependent targets in CSCs, with well-established master CSC regulators represented in the target geneset. ${ }^{82}$ This novel nuclear PKC- $\theta$ epigenetic mechanism in human CSCs shows that active nuclear PKC- $\theta$ is critical for mediating open/ permissive chromatin regulatory regions and is essential for the transcription of key CSC-enriched genes. Our findings show that targeting PKC- $\theta$ with selective inhibitors such as C27 or siRNA abrogates breast CSCs. Furthermore, dualspecificity phosphatases (DUSPs), which dephosphorylate

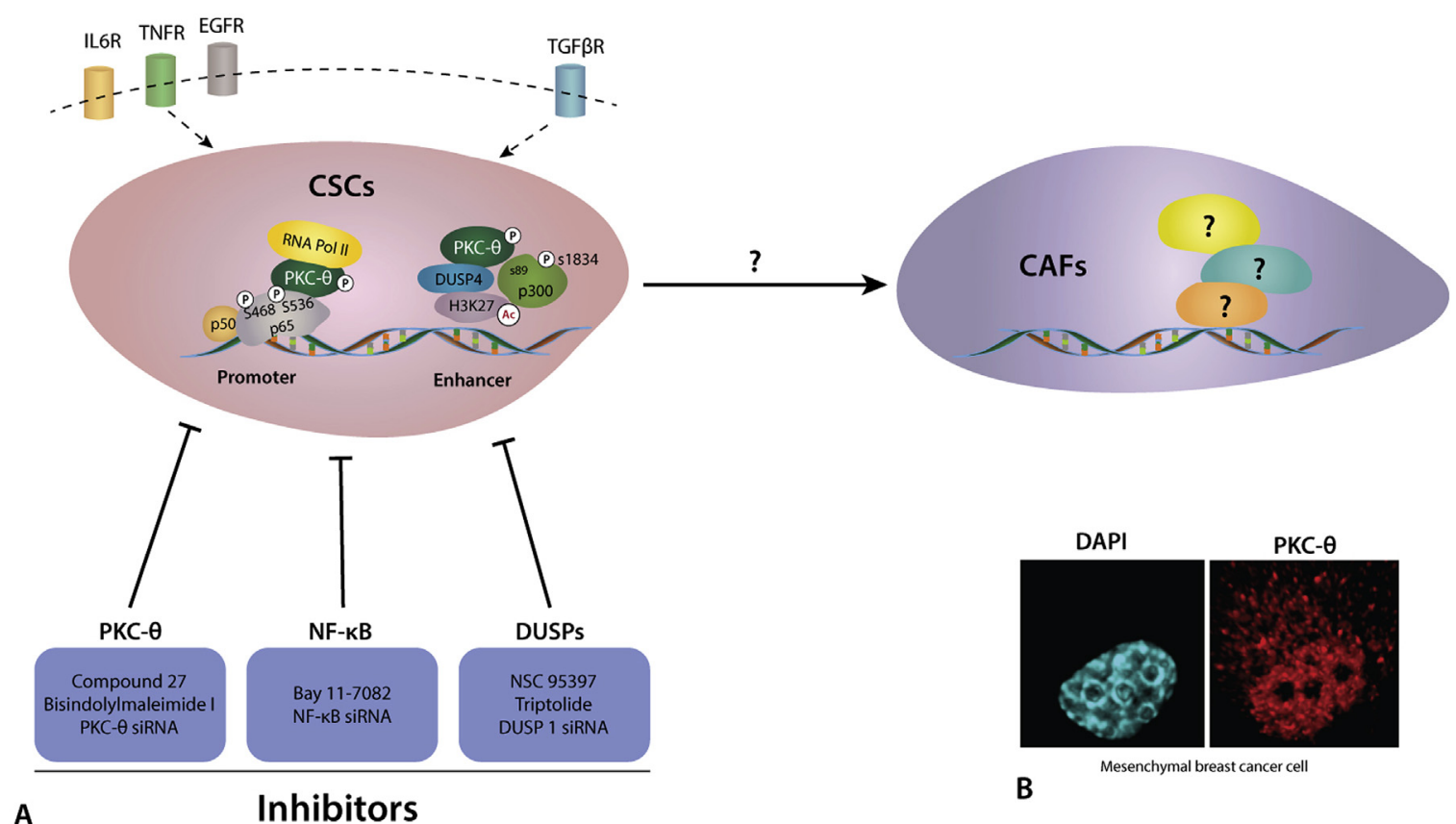

Fig. 5 New classes of epigenetic therapeutic targets in breast cancer. (A) The role of PKC-theta (PKC- $\theta$ ), DUSPs, and NF- $\mathrm{BB}$ in the biology of cancer stem cells (CSCs) in the context of chromatin-tethered kinases and epigenetic enzymes. Previous research from the Rao Laboratory demonstrated that, in the mesenchymal state, activation of PKC- $\theta$ via TGF- $\beta$ and inflammatory signals (e.g., IL6, TNF- $\alpha$ and EGF) phosphorylates NF- $\kappa$ B (p50:p65) for translocation to the nucleus, where NF- $\kappa B$ tethers PKC$\theta$ to the chromatin template to recruit RNA polymerase II (RNA POL II) and subsequent transcription of CSC-inducible genes. In addition, together with PKC- $\theta$, DUSP activation has been shown to have a critical role in the dephosphorylation of P300 at serine 89, which can further activate $\mathrm{H} 3 \mathrm{~K} 27$ acetylation at CSC repressive gene enhancers. These key molecular mechanisms can be inhibited (see blue inhibitor boxes) by blocking the epigenetic kinase PKC- $\theta$ [with compound 27 (C27), bisindolylmaleimide I (BIM), or PKC- $\theta$ siRNA] or NF- $\kappa$ B (with Bay11-7082 or NF- $\kappa$ B p50/p65 siRNA) or DUSP1 (with NSC 95397, triptolide, or DUSP1 siRNA). (B) High resolution $3 \mathrm{D}$ image of a mesenchymal breast cancer cell, clearly displaying the nuclear bias of the epigenetic kinase PKC- $\theta$ in this cell. 
threonine/serine and tyrosine residues on their substrates, are involved in EMT and breast CSC regulation and are induced during EMT in a PKC pathway signal-mediated EMT model. ${ }^{83}$ Hence, given that CSCs mediate the transition to CAFs in breast cancer, we postulate that targeting this novel PKC-epigenetic axis may offer new therapeutic avenues to simultaneously eliminate CAFs and CSCs in breast cancer. Given the importance of TGF- $\beta$ and NF- $\kappa \mathrm{B}$ in CAF-mediated tumorigenesis, ${ }^{29,87}$ PKC- $\theta$ may also have a direct epigenetic role in CAFs that needs to be elucidated. PKCs belong to a family of 11 isoforms (conventional, atypical, and novel), and the interplay between these kinases, CAFs, and CSCs also requires further investigation. It remains to be seen if therapeutic targeting of these enzymes will abrogate CAFs in breast cancer in vivo.

Therefore, many components of the TME, particularly those related to EMT activation in breast cancer cells and the possible entry of these cells into the CSC state, ${ }^{5}$ could potentially be targeted for cancer prevention and treatment as part of epi-drug development. Treatments that normalise the stroma are also potentially powerful therapeutic strategies. ${ }^{88}$

\section{CONCLUSIONS}

This review highlights that research into breast cancer development, progression, and management can no longer focus solely on malignant breast epithelial cells but also needs to address the role of the TME both in the primary cancer and in metastases. The role of the TME now needs to be considered when assessing the impact of various treatments. In the future, pathologists may need to map specific epigenetic profiles of individual TME components as part of the prognostic and predictive work-up of an individual cancer and to guide precision therapy.

Conflicts of interest and sources of funding: The original research presented in this review was supported under NHMRC Projects funding scheme (project number APP1048065). The authors state there are no conflicts of interest to disclose.

Address for correspondence: Sudha Rao, Health Research Institute, Faculty of Education, Science, Technology and Mathematics, University of Canberra, Bruce, ACT 2607, Australia. E-mail: sudha.rao@ canberra.edu.au Address for correspondence: Jane Dahlstrom, Anatomical Pathology, ACT Pathology and Australian National University Medical School, The Canberra Hospital, PO Box 11, Woden, ACT 2606, Australia. E-mail: Jane. dahlstrom@act.gov.au

\section{References}

1. Jin B, Li Y, Robertson KD. DNA Methylation: superior or subordinate in the epigenetic hierarchy? Genes Cancer 2011; 2: 607-17.

2. Nightingale KP, O'Neill LP, Turner BM. Histone modifications: signalling receptors and potential elements of a heritable epigenetic code. Curr Opin Genet Dev 2006; 16: 125-36.

3. Weber CM, Henikoff S. Histone variants: dynamic punctuation in transcription. Genes Dev 2014; 28: 672-82.

4. Birgisdottir V, Stefansson OA, Bodvarsdottir SK, et al. Epigenetic silencing and deletion of the BRCA1 gene in sporadic breast cancer. Breast Cancer Res 2006; 8: R38.

5. Pattabiraman DR, Weinberg RA. Tackling the cancer stem cells - what challenges do they pose? Nat Rev Drug Discov 2014; 13: 497-512.

6. Semenza GL. The hypoxic tumor microenvironment: A driving force for breast cancer progression. Biochim Biophys Acta 2016; 1863: 382-91.

7. Simmons A, Burrage PM, Nicolau Jr DV, Lakhani SR, Burrage K. Environmental factors in breast cancer invasion: a mathematical modelling review. Pathology 2017; 49: 172-80.
8. Elenbaas B, Weinberg RA. Heterotypic signaling between epithelial tumor cells and fibroblasts in carcinoma formation. Exp Cell Res 2001; 264: $169-84$.

9. Yamamura Y, Asai N, Enomoto A, et al. Akt-Girdin signaling in cancer-associated fibroblasts contributes to tumor progression. Cancer Res 2015; 75: 813-23.

10. Buchsbaum RJ, Oh SY. Breast cancer-associated fibroblasts: where we are and where we need to go. Cancers 2016; 8: E19.

11. Tyan SW, Kuo WH, Huang CK, et al. Breast cancer cells induce cancerassociated fibroblasts to secrete hepatocyte growth factor to enhance breast tumorigenesis. PloS One 2011; 6: e15313.

12. Martinez-Outschoorn UE, Pavlides S, Whitaker-Menezes D, et al. Tumor cells induce the cancer associated fibroblast phenotype via caveolin-1 degradation: implications for breast cancer and DCIS therapy with autophagy inhibitors. Cell Cycle 2010; 9: 2423-33.

13. Orimo A, Weinberg RA. Heterogeneity of stromal fibroblasts in tumors. Cancer Biol Ther 2007; 6: 618-9.

14. Qiao A, Gu F, Guo X, et al. Breast cancer-associated fibroblasts: their roles in tumor initiation, progression and clinical applications. Front Med 2016; 10: 33-40.

15. Tchou J, Kossenkov AV, Chang L, et al. Human breast cancer associated fibroblasts exhibit subtype specific gene expression profiles. BMC Med Genomics 2012; 5: 39.

16. Singer CF, Gschwantler-Kaulich D, Fink-Retter A, et al. Differential gene expression profile in breast cancer-derived stromal fibroblasts. $B$ Cancer Res Treat 2008; 110: 273-81.

17. Xing F, Saidou J, Watabe K. Cancer associated fibroblasts (CAFs) in tumor microenvironment. Front Biosci 2010; 15: 166-79.

18. Fiegl H, Millinger S, Goebel G, et al. Breast cancer DNA methylation profiles in cancer cells and tumor stroma: association with HER-2/neu status in primary breast cancer. Cancer Res 2006; 66: 29-33.

19. Hu M, Yao J, Cai L, et al. Distinct epigenetic changes in the stromal cells of breast cancers. Nat Genet 2005; 37: 899-905.

20. Mao Y, Keller ET, Garfield DH, et al. Stromal cells in tumor microenvironment and breast cancer. Cancer Metastasis Rev 2013; 32: 303-15.

21. Tang X, Hou Y, Yang G, et al. Stromal miR-200s contribute to breast cancer cell invasion through CAF activation and ECM remodeling. Cell Death Differ 2016; 23: 132-45.

22. Scheel C, Weinberg RA. Cancer stem cells and epithelial-mesenchymal transition: concepts and molecular links. Semin Cancer Biol 2012; 22: 396-403.

23. Soon PS, Kim E, Pon CK, et al. Breast cancer-associated fibroblasts induce epithelial-to-mesenchymal transition in breast cancer cells Endocr Relat Cancer 2013; 20: 1-12.

24. Angelucci C, Maulucci G, Lama G, et al. Epithelial-stromal interactions in human breast cancer: effects on adhesion, plasma membrane fluidity and migration speed and directness. PloS One 2012; 7 : e50804.

25. Yu Y, Xiao CH, Tan LD, et al. Cancer-associated fibroblasts induce epithelial-mesenchymal transition of breast cancer cells through paracrine TGF-beta signalling. Br J Cancer 2014; 110: 724-32.

26. Chaffer CL, Marjanovic ND, Lee T, et al. Poised chromatin at the ZEB1 promoter enables breast cancer cell plasticity and enhances tumorigenicity. Cell 2013; 154: 61-74.

27. Tsuyada A, Chow A, Wu J, et al. CCL2 mediates cross-talk between cancer cells and stromal fibroblasts that regulates breast cancer stem cells. Cancer Res 2012; 72: 2768-79.

28. De Marco P, Lappano R, Francesco EM, et al. GPER signalling in both cancer-associated fibroblasts and breast cancer cells mediates a feedforward IL1beta/IL1R1 response. Sci Rep 2016; 6: 24354.

29. Erez N, Truitt M, Olson P, et al. Cancer-associated fibroblasts are activated in incipient neoplasia to orchestrate tumor-promoting inflammation in an NF-kappaB-dependent manner. Cancer Cell 2010; 17 $135-47$.

30. Krtolica A, Parrinello S, Lockett S, et al. Senescent fibroblasts promote epithelial cell growth and tumorigenesis: a link between cancer and aging. Proc Natl Acad Sci USA 2001; 98: 12072-7.

31. Kuperwasser C, Chavarria T, Wu M, et al. Reconstruction of functionally normal and malignant human breast tissues in mice. Proc Natl Acad Sci USA 2004; 101: 4966-71.

32. Palmieri C, Roberts-Clark D, Assadi-Sabet A, et al. Fibroblast growth factor 7 , secreted by breast fibroblasts, is an interleukin-1beta-induced paracrine growth factor for human breast cells. J Endocrinol 2003; 177: $65-81$.

33. Scherz-Shouval R, Santagata S, Mendillo ML, et al. The reprogramming of tumor stroma by HSF1 is a potent enabler of malignancy. Cell 2014; 158: $564-78$.

34. Junttila MR, de Sauvage FJ. Influence of tumour micro-environment heterogeneity on therapeutic response. Nature 2013; 501: 346-54.

35. Narunsky L, Oren R, Bochner F, et al. Imaging aspects of the tumor stroma with therapeutic implications. Pharmacol Ther 2014; 141: 192-208. 
36. Liao D, Luo Y, Markowitz D, et al. Cancer associated fibroblasts promote tumor growth and metastasis by modulating the tumor immune microenvironment in a 4T1 murine breast cancer model. PloS One 2009; 4: e7965.

37. Bifulco M, Pisanti S. "Adiponcosis": a new term to name the obesity and cancer link. J Clinical Endocrinol Metab 2013; 98: 4664-5.

38. D'Esposito V, Liguoro D, Ambrosio MR, et al. Adipose microenvironment promotes triple negative breast cancer cell invasiveness and dissemination by producing CCL5. Oncotarget 2016; 7: 24495-509.

39. Wolfson B, Eades G, Zhou Q. Adipocyte activation of cancer stem cell signaling in breast cancer. World J Biol Chem 2015; 6: 39-47.

40. Dirat B, Bochet L, Dabek M, et al. Cancer-associated adipocytes exhibit an activated phenotype and contribute to breast cancer invasion. Cancer Res 2011; 71: 2455-65.

41. Lee E, Pandey NB, Popel AS. Crosstalk between cancer cells and blooc endothelial and lymphatic endothelial cells in tumour and organ microenvironment. Exp Rev Mol Med 2015; 17: e3.

42. Lee E, Fertig EJ, Jin K, et al. Breast cancer cells condition lymphatic endothelial cells within pre-metastatic niches to promote metastasis. Nat Commun 2014; 5: 4715.

43. Dushyanthen S, Beavis PA, Savas P, et al. Relevance of tumorinfiltrating lymphocytes in breast cancer. BMC Med 2015; 13: 202.

44. Luen SJ, Savas P, Fox SB, Salgado R, Loi S. Tumour-infiltrating lymphocytes and the emerging role of immunotherapy in breast cancer. Pathology 2017; 49: 141-55.

45. Markowitz J, Wesolowski R, Papenfuss T, et al. Myeloid-derived sup pressor cells in breast cancer. Br Cancer Res Treat 2013; 140: 13-21.

46. Mamessier E, Sylvain A, Thibult ML, et al. Human breast cancer cells enhance self tolerance by promoting evasion from NK cell antitumor immunity. J Clin Invest 2011; 121: 3609-22.

47. Theocharis AD, Skandalis SS, Neill T, et al. Insights into the key roles of proteoglycans in breast cancer biology and translational medicine. Biochim Biophys Acta 2015; 1855: 276-300.

48. Chernov AV, Strongin AY. Epigenetic regulation of matrix metal loproteinases and their collagen substrates in cancer. Biomol Concepts 2011; 2: 135-47.

49. Chernov AV, Baranovskaya S, Golubkov VS, et al. Microarray-based transcriptional and epigenetic profiling of matrix metalloproteinases, collagens, and related genes in cancer. J Biol Chem 2010; 285: 19647-59.

50. Nguyen DX, Bos PD, Massagué J. Metastasis: from dissemination to organ-specific colonization. Nat Rev Cancer 2009; 9: 274-84.

51. Psaila B, Lyden D. The metastatic niche: adapting the foreign soil. Nat Rev Cancer 2009; 9: 285-93.

52. Ursini-Siegel J, Siegel PM. The influence of the pre-metastatic niche on breast cancer metastasis, Cancer Lett 2015; 380: 281 -8.

53. Wels J, Kaplan RN, Rafii S, Lyden D. Migratory neighbors and distan invaders: tumor-associated niche cells. Genes Devel 2008; 22: 559-74.

54. Malanchi I, Santamaria-Martínez A, Susanto E, et al. Interactions between cancer stem cells and their niche govern metastatic colonization. Nature 2012; 481: 85-9.

55. Zhang L, Zhang S, Yao J, et al. Microenvironment-induced PTEN loss by exosomal microRNA primes brain metastasis outgrowth. Nature 2015; 527: 100-4

56. Karagiannis GS, Poutahidis T, Erdman SE, Kirsch R, Riddell RH, Diamandis EP. Cancer-associated fibroblasts drive the progression of metastasis through both paracrine and mechanical pressure on cancer tissue. Mol Cancer Res 2012; 10: 1403-18.

57. Brentnall TA, Lai LA, Coleman J, Bronner MP, Pan S, Chen R. Arousal of cancer-associated stroma: overexpression of palladin activates fibroblasts to promote tumor invasion. PLoS One 2012; 7: e30219.

58. Gaggioli C, Hooper S, Hidalgo-Carcedo C, et al. Fibroblast-led col lective invasion of carcinoma cells with differing roles for RhoGTPases in leading and following cells. Nat Cell Biol 2007; 9: 1392-400.

59. Quail DF, Joyce JA. Microenvironmental regulation of tumor progression and metastasis. Nat Med 2013; 19: 1423-37.

60. Wendler F, Favicchio R, Simon T, Alifrangis C, Stebbing J, Giamas G Extracellular vesicles swarm the cancer microenvironment: from tumor-stroma communication to drug intervention. Oncogene 2016; Aug 22: (Epub ahead of print). http://dx.doi.org/10.1038/onc.2016.253.

61. Chowdhury R, Webber JP, Gurney M, Mason MD, Tabi Z, Clayton A Cancer exosomes trigger mesenchymal stem cell differentiation into proangiogenic and pro-invasive myofibroblasts. Oncotarget 2015; 6 : $715-31$.

62. Cho JA, Park H, Lim EH, Lee KW. Exosomes from breast cancer cells can convert adipose tissue-derived mesenchymal stem cells into myofibroblast-like cells. Int J Oncol 2012; 40: 130-8.
63. Chen Y, Wang L, Zhu Y, et al. Breast cancer resistance protein (BCRP) containing circulating microvesicles contribute to chemoresistance in breast cancer. Oncol Lett 2015; 10: 3742-8.

64. Cai Z, Yang F, Yu L, et al. Activated T cell exosomes promote tumor invasion via Fas signaling pathway. J Immunol 2012; 188: 5954-61.

65. Le MT, Hamar P, Guo C, et al. miR-200-containing extracellula vesicles promote breast cancer cell metastasis. J Clin Invest 2014; 124 5109-28.

66. Shekhar MP, Santner S, Carolin KA, et al. Direct involvement of breast tumor fibroblasts in the modulation of tamoxifen sensitivity. Am Pathol 2007; 170: 1546-60.

67. Pontiggia O, Sampayo R, Raffo D, et al. The tumor microenvironment modulates tamoxifen resistance in breast cancer: a role for soluble stromal factors and fibronectin through betal integrin. $\mathrm{Br}$ Cancer Res Treat 2012; 133: 459-71.

68. Luo H, Yang G, Yu T, et al. GPER-mediated proliferation and estradiol production in breast cancer-associated fibroblasts. Endocr Relat Cancer 2014; 21: 355-69.

69. Yuan J, Liu M, Yang L, et al. Acquisition of epithelial-mesenchymal transition phenotype in the tamoxifen-resistant breast cancer cell: a new role for $\mathrm{G}$ protein-coupled estrogen receptor in mediating tamoxifen resistance through cancer-associated fibroblast-derived fibronectin and beta1-integrin signaling pathway in tumor cells. Br Cancer Res 2015; 17: 69 .

70. Mao Y, Zhang Y, Qu Q, et al. Cancer-associated fibroblasts induce trastuzumab resistance in HER2 positive breast cancer cells. Mol Biosyst 2015; 11: 1029-40.

71. Farmer P, Bonnefoi H, Anderle P, et al. A stroma-related gene signature predicts resistance to neoadjuvant chemotherapy in breast cancer. Nat Med 2009; 15: 68-74.

72. Barker HE, Paget JT, Khan AA, et al. The tumour microenvironment after radiotherapy: mechanisms of resistance and recurrence. Nat Rev Cancer 2015; 15: 409-25.

73. Kelderman S, Schumacher TN, Haanen JB. Acquired and intrinsic resistance in cancer immunotherapy. Mol Oncol 2014; 8: 1132-9.

74. Kouzarides T. SnapShot: histone-modifying enzymes. Cell 2007; 131: 822.

75. Falahi F, van Kruchten M, Martinet N, et al. Current and upcoming approaches to exploit the reversibility of epigenetic mutations in breast cancer. Br Cancer Res 2014; 16: 412.

76. Mottamal M, Zheng S, Huang TL, et al. Histone deacetylase inhibitors in clinical studies as templates for new anticancer agents. Molecules 2015; 20: 3898-941.

77. Mataga MA, Rosenthal S, Heerboth S, et al. Anti-breast cancer effects of histone deacetylase inhibitors and calpain inhibitor. Anticancer Re 2012; 32: 2523-9.

78. Billam M, Sobolewski MD, Davidson NE. Effects of a novel DNA methyltransferase inhibitor zebularine on human breast cancer cells. $\mathrm{Br}$ Cancer Res Treat 2010; 120: 581-92.

79. Nebbioso A, Carafa V, Benedetti R, et al. Trials with 'epigenetic' drugs: an update. Mol Oncol 2012; 6: 657-82.

80. Kim K, Skora AD, Li Z, et al. Eradication of metastatic mouse cancers resistant to immune checkpoint blockade by suppression of myeloidderived cells. Proc Natl Acad Sci USA 2014; 111: 11774-9.

81. Wrangle J, Wang W, Koch A, et al. Alterations of immune response of non-small cell lung cancer with azacytidine. Oncotarget 2013; 4: $2067-79$.

82. Zafar A, Wu F, Hardy K, et al Chromatinized protein kinase C-theta directly regulates inducible genes in epithelial to mesenchymal transition and breast cancer stem cells. Mol Cell Biol 2014; 34: 2961-80.

83. Boulding T, Wu F, McCuaig R, et al. Differential roles for DUSP family members in epithelial-to-mesenchymal transition and cancer stem cell regulation in breast cancer. PloS One 2016; 11: e0148065.

84. Phillips TM, McBride WH, Pajonk F. The response of CD24(-/low)/ CD44+ breast cancer-initiating cells to radiation. J Natl Cancer Inst 2006; 98: 1777-85.

85. Rao S, Procko E, Shannon MF. Chromatin remodeling, measured by a novel real-time polymerase chain reaction assay, across the proxima promoter region of the IL-2 gene. J Immunol 2001; 167: 4494-503.

86. Strahl BD, Allis CD. The language of covalent histone modifications. Nature 2000; 403: 41-5.

87. Tang B, Vu M, Booker T, et al. TGF-beta switches from tumor suppressor to prometastatic factor in a model of breast cancer progression. $J$ Clin Invest 2003; 112: 1116-24.

88. Mueller MM, Fusenig NE. Friends or foes - bipolar effects of the tumour stroma in cancer. Nat Rev Cancer 2004; 4: 839-49. 